\title{
Correction to: Efficient photodynamic therapy against drug-resistant prostate cancer using replication-deficient virus particles and talaporfin sodium
}

\author{
Sharmin Akter ${ }^{1,2} \cdot$ Sachiko Saito ${ }^{1} \cdot$ Mizuho Inai $^{3} \cdot$ Norihiro Honda $^{1,4} \cdot$ Hisanao Hazama $^{1} \cdot$ Tomoyuki Nishikawa $^{5}$. \\ Yasufumi Kaneda ${ }^{5}$ Kunio Awazu ${ }^{1,3,6}$
}

Published online: 13 July 2020

(C) Springer-Verlag London Ltd., part of Springer Nature 2020

\section{Correction to: Lasers in Medical Science} https://doi.org/10.1007/s10103-020-03076-1

After publication of this paper, the authors determined an error in Fig. 1. Below is the correct Fig. 1.

Publisher's note Springer Nature remains neutral with regard to jurisdictional claims in published maps and institutional affiliations.

The online version of the original article can be found at https://doi.org/ 10.1007/s10103-020-03076-1

Sharmin Akter

akter-s@see.eng.osaka-u.ac.jp; sharmin.akter@bau.edu.bd

1 Graduate School of Engineering, Osaka University, 2-1 Yamadaoka, Suita, Osaka 565-0871, Japan

2 Department of Physiology, Bangladesh Agricultural University, Mymensingh 2202, Bangladesh

3 Graduate School of Frontier Biosciences, Osaka University, 1-1 Yamadaoka, Suita, Osaka 565-0871, Japan

4 Institute for Academic Initiatives, OsakaUniversity, 1-1 Yamadaoka, Suita, Osaka 565-0871, Japan

5 Graduate School of Medicine, Osaka University, 2-2 Yamadaoka, Suita, Osaka 565-0871, Japan

6 Global Center for Medical Engineering and Informatics, Osaka University, 2-2 Yamadaoka, Suita, Osaka 565-0871, Japan

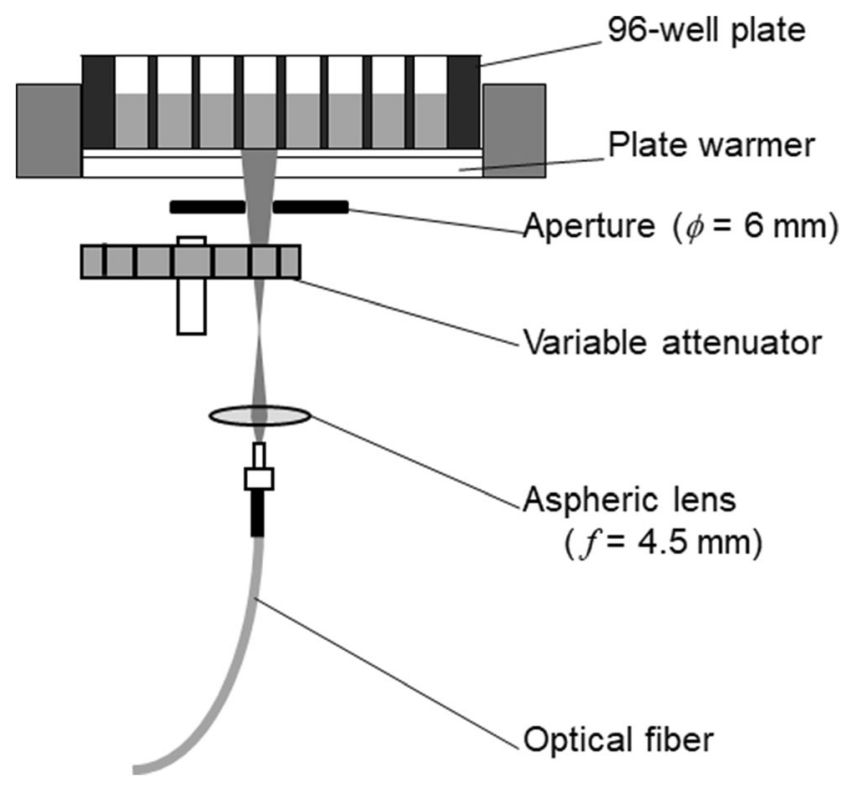

Fig. 1 Schematic diagram of the optical system for laser irradiation, where $\phi$ and $f$ indicate the diameter and focal length, respectively. Light was guided through an optical fiber and the exit surface of the fiber was projected on the bottom of the 96-well cell culture plate by an aspheric lens. Power density was adjusted by the variable attenuator. The position of the cell culture plate was controlled by a two-axis motorized linear stage. The temperature of the well plate was kept at $37{ }^{\circ} \mathrm{C}$ using a plate warmer 